\title{
Modelling generalized firms' restructuring using inverse DEA
}

\author{
Gholam R. Amin \\ Faculty of Business, University of New Brunswick at Saint John, \\ Saint John, NB, E2L 4L5, Canada \\ Email: gamin@unb.ca
}

\author{
Ali Emrouznejad \\ Professor in Business Analytics \\ Operations \& Information Management Group \\ Aston Business School \\ Aston University, \\ Birmingham, \\ UK \\ Email:a.emrouznejad@aston.ac.uk \\ Web: www.DEAzone.com

\section{Said Gattoufi} \\ Laboratoire SOIE \\ Institut Supérieur de Gestion de Tunis \\ Université de Tunis \\ Tunisie
}




\title{
Modelling generalized firms' restructuring using inverse DEA
}

\author{
Gholam R. Amina, Ali Emrouznejad ${ }^{\mathrm{b} 1}$, Said Gattoufic, \\ ${ }^{a}$ Faculty of Business, University of New Brunswick at Saint John, Saint John, NB, E2L 4L5, Canada \\ ${ }^{b}$ Operations \& Information Management Group, Aston Business School, Aston University, Birmingham, UK \\ c Laboratoire SOIE, Institut Supérieur de Gestion, Université de Tunis, Tunisie
}

\begin{abstract}
The key consideration for firms' restructuring is improving their operational efficiencies. Market conditions often offer opportunities or generate threats that can be handled by restructuring scenarios through consolidation, to create synergy, or through split, to create reverse synergy. A generalized restructuring refers to a move in a business market where a homogeneous set of firms, a set of pre-restructuring decision making units (DMUs), proceed with a restructuring to produce a new set of post-restructuring entities in the same market to realize efficiency targets. This paper aims to develop a novel inverse Data Envelopment Analysis based methodology, called GInvDEA (Generalized Inverse DEA), for modeling the generalized restructuring. Moreover, the paper suggests a linear programming model that allows determining the lowest performance levels, measured by efficiency that can be achieved through a given generalized restructuring. An application in banking operations illustrates the theory developed in the paper.
\end{abstract}

Keywords: Generalized restructuring; Consolidation; Split; Efficiency; Data Envelopment Analysis (DEA); Inverse DEA.

\section{Introduction}

The business environment of firms is often characterized by exceptional conditions that either offer opportunities for synergies through mergers/acquisitions or reverse

\footnotetext{
${ }^{1}$ Corresponding author:
}

Ali Emrouznejad, Professor in Business Analytics, Operations \& Information Management Group, Aston Business School, Aston University, Birmingham, UK,

Web: www.DEAzone.com Email: a.emrouznejad@aston.ac.uk 
synergies through split. Both moves are considered as restructuring. The common form of merger happens when two firms combine their activities to create a new merged entity intended to perform better. Reversely, the restructuring for a better performance can take the form of downsizing by splitting the firm to create new entities dedicated to the inherited activities.

Any restructuring decision is usually accompanied with a predefined performance target to be reached (Wu et al., 2014, Gaughan, 2010; Gattoufi et al. 2014). The assessment of the potential gains, compared with the predefined target, has been discussed in the Data Envelopment Analysis (DEA) literature, benefiting from the ability of DEA modeling to assess relative efficiency. The DEA literature was highly enriched by studies that discussed the importance of firms' consolidation, mostly for the cases of Mergers and Acquisitions (M\&A), and the gains generated for the firms as well as for their stakeholders. These studies cover a wide range of applications in mergers including healthcare (Harris et al., 2000; Ferrier and Valdmanis, 2004; Kristensen et al., 2010; Leleu et al., 2012), telecommunications (Liu et al., 2007), forestry (Bogetoft et al., 2003), agriculture (Bogetoft and Wang, 2005), electricity (Kwoka and Pollitt, 2010), airlines (Kong et al., 2012), water sector (De Witte and Dijkgraaf, 2010), and banking (Avkiran, 1999; Wu and Birge, 2011; Halkos and Tzeremes; 2013).

Some of these studies focused on the assessment of the potential gains that can be obtained through a merger, compared to what the firms realize individually, using a variety of DEA models, like Avkiran (1999), Bogetoft et al. (2003), Bogetoft and Wang (2005), Kristensen et al. (2010), Lozano and Villa (2010), Wu et al. (2011), and Peyrache (2013). However, for the best knowledge of the authors, none of the studies considered the case of split or the general case of mergers when two or more entities are generated by the restructuring operation.

Despite its usefulness as an analytical tool to evaluate different alternatives, conventional DEA approach does not allow determining levels of production factors of a firm for a given efficiency score. Unlike the conventional DEA, where the 
objective is to calculate the efficiency score of a specific decision making unit (DMU), Inverse DEA (InvDEA) assumes efficiency as a given parameter, that can be an efficiency level predefined as a strategic target, and aims to compute the quantities of inputs and outputs that are required to achieve a pre-specified efficiency level.

The idea of the inverse DEA first appeared in Zhang and Cui (1999), though the inverse DEA was formally studied at first in Wei et al. (2000) and subsequently discussed in Yan et al. (2002), and Frija et al. (2011). In Zhang and Cui (1999) the input increases of a DMU are estimated for its given output increases under the constant returns to scale efficiency-fixed constraints. Subsequently, the literature on inverse DEA has been extended and this problem has been studied in many theoretical and applied publications, including Hadi-Vencheh et al. (2008), HadiVencheh and Foroughi (2006), Jahanshahloo et al. (2004a, 2004b, 2005), and Lin (2010). Further to this, Lertworasirikul et al. (2011) and Ghiyasi (2015) proposed an inverse variable returns to scale model for a resource allocation problem. HadiVencheh et al. (2015) presented an inverse DEA model in the presence of imprecise data. In addition, Jahanshahloo et al. (2015) studied an inverse DEA application for the systems with inter-temporal dependence. A review and extension to the inverse DEA method and applications introduced in Ghobadi and Jahangiri (2015). More recently, Zhang and Cui (2016) presented an extension of the inverse DEA model and Lim (2016) introduced an inverse DEA model with the frontier change for setting a new product target.

Gattoufi et al. (2014) proposed a methodology to identify the required reduction in inputs, or increase in outputs depending on whether the analysis is input or output oriented, in order to realize a predefined efficiency target for a merger between at least two merging DMUs producing a single merged DMU. However, generality imposes two forms of restructuring namely consolidation and split. On the one hand, synergy can be obtained through a consolidation that takes place when a given group of DMUs merge producing at least two merged entities. On the other 
hand, reverse synergy is obtained by a set of firms splitting their activities into a larger number of dedicated new entities.

One of the important cases that has been neglected in the merging and acquisition literature, using DEA or any other methodology for efficiency assessment and analysis, is the situation where a set of DMUs (three or more) are subject of restructuring to generate a different set of DMUs (two or more). An example of this is merging Lloyds Bank, TSB Bank and Cheltenham \& Gloucester to form Lloyds TSB Bank which in 2013 has been restructured to two banks: Lloyds bank and TSB Bank.

The current paper extends the state-of-the-art in the area of firm restructuring and enriches it with new concepts and analytical tools. It introduces a novel method for handling generalized restructuring situations using inverse DEA, called GInvDEA. GInvDEA is capable of suggesting the simultaneous redistribution of multiple inputs and multiple outputs inherited from pre-restructuring DMUs between postrestructuring DMUs. The redistribution is made in a way that allows the merged entities to achieve their desired predefined efficiency targets. The paper also suggests a model for finding the lowest value of efficiency targets the postrestructuring DMUs can realize. Such a solution can be informative since it provides foresight about the post-restructuring market positioning of the restructured DMUs and the minimum efficiency scores they can achieve. The decision of restructuring is hence encouraged as long as the lowest bounds are judged satisfactory. Illustration of the proposed GInvDEA methodology is through an application in banking, covering both consolidation and split generalized restructuring cases.

It would be intuitive noting that the main contribution of the classical DEA models in mergers and acquisitions is estimating the potential gains from mergers. This simply means the potential achievement that each involved DMU can attain through a merger. For instance, assume DMU-A could save $\$ 100$ by reducing its inputs guided by a standard input-oriented DEA model and similarly DMU-B could save $\$ 200$. If these DMUs could save $\$ 350$ by reducing their inputs after merging together, then $\$ 50$ would be additional saving that is gained by the merger. This is the main 
question that is answered by the standard DEA models used in the literature of mergers and acquisitions, see Bogetoft and Wang (2005). More precisely, the classical DEA models provide a way for policy-makers to pre-evaluate the potential gains from possible mergers and acquisitions (Halkos et al. 2015). This paper, on the other hand, proposes a generalized InvDEA methodology that answers the following main question. If a group of $p(p \geq 3)$ pre-merger DMUs decide to go through a merger for generating a group of $q(p>q \geq 2)$ post-merger DMUs that are able to achieve given efficiency targets, then how much inputs (outputs) they would need to use (to produce) for securing the specified level of efficiency after merging? To the best of our knowledge, this has not been addressed in the DEA literature.

The rest of this paper unfolds in five sections. Section 2 presents the InvDEA method suggested to address the standard merger case producing a single merged DMU. Section 3 defines a generalized restructuring model, the GInvDEA, which addresses the situation where multiple DMUs are subject of restructuring to generate multiple DMUs with predefined efficiency targets. Section 4 proposes a linear programming model that allows determining the minimum performance that can be achieved through a generalized restructuring scenario. An application in banking is used in Section 5 to illustrate the generalized restructuring theory developed throughout the paper. Finally, concluding remarks and directions for future research are given in Section 6.

\section{InvDEA for merger}

The mathematical modeling suggested for merger by the InvDEA approach (Gattoufi et al. 2014) is presented hereafter. It allows determining the levels of inputs and outputs for a single merged firm, following a merger between at least two firms. Suppose that we have $n$ DMUs where the $j^{\text {th }}$ unit uses $m$ inputs $x_{i j}$ in order to produce $s$ outputs $y_{r j}$, for all $r=1, \ldots, s, i=1, \ldots, m$ and $j=1, \ldots, n$. Assume that two DMUs, $k$ and $l$, are consolidating their activities to create a single merged DMU, 
namely $\mathrm{M}$. That is, both merging units are disappearing to generate a new merged unit. Also, let $\mathrm{T}$ indicates the set of indices of all DMUs except $k$ and $l$. The corresponding input-oriented InvDEA model is as follows:

$$
\begin{aligned}
& \min \sum_{i=1}^{m}\left(\alpha_{i k}+\alpha_{i l}\right) \\
& \text { s.t. } \\
& \qquad \sum_{j \in T} x_{i j} \lambda_{j}+\left(\alpha_{i k}+\alpha_{i l}\right) \lambda_{M}-\left(\alpha_{i k}+\alpha_{i l}\right) \bar{\theta} \leq 0 \quad i=1, \ldots, m \\
& \sum_{j \in T} y_{r j} \lambda_{j}+\left(y_{r k}+y_{r l}\right) \lambda_{M} \geq\left(y_{r k}+y_{r l}\right) \quad r=1, \ldots, s \\
& \sum_{j \in T} \lambda_{j}+\lambda_{M}=1 \\
& 0 \leq \alpha_{i k} \leq x_{i k} \quad i=1, \ldots, m \\
& 0 \leq \alpha_{i l} \leq x_{i l} \quad i=1, \ldots, m \\
& \quad \lambda_{j} \geq 0 \forall j \in T, \lambda_{M} \geq 0
\end{aligned}
$$

where, $\alpha_{i k}$ and $\alpha_{i l}$ are respectively the quantities of the $i^{\text {th }}$ input $(i=1, \ldots, m)$ inherited by the merged unit that should be kept from merging DMUs $k$ and $l$. Moreover, $\bar{\theta}$ is the predefined desired efficiency target for the merged entity $M$. The objective of the above model is to determine the minimum quantity of inputs inherited from the merging DMUs required for $\mathrm{M}$ to realize $\bar{\theta}$. The above nonlinear programming (NLP) model is then linearized and solved to determine the maximum possible reduction in the inherited inputs usage. The generalized methodology suggested here deals with firms' restructuring in general, where several prerestructuring DMUs can be considered for generating several post-restructuring units. Two cases are to be distinguished in presenting and discussing the generalized restructuring InvDEA methodology, namely the case of consolidation and that of a split.

\section{Modelling generalized restructuring}


We define a generalized restructuring as a process that considers a set of $\bar{p}$ selected pre-restructuring DMUs, indexed in $P$, to produce $\bar{q}$ post- restructuring DMUs, indexed in $Q$, where $\min \{\bar{p}, \bar{q}\} \geq 2$. Moreover, a restructuring is defined as a consolidation if $\bar{p} \geq \bar{q}+1$, the number of pre-restructuring DMUs is at least one more DMU than post-restructuring DMUs. Alternatively, if $\bar{q} \geq \bar{p}+1$ then the corresponding restructuring is called a split. The special case of $\bar{p}=\bar{q}$ is a pure restructuring where the pre-restructuring DMUs survive with radical changes in their activities and/or their ownership structure through swap or acquisition or any other form. The generalization in this paper resides in the fact that, unlike a merger where a number of merging DMUs produces a single merged DMU, there are no restrictions relating the pre-restructuring number of DMUs to that of the postrestructuring. The models suggested hereafter, denoted GInvDEA, address both consolidation and split simultaneously.

Let $I$ and $R$ be respectively the sets of indices for inputs and outputs. Let $\bar{P}$ be the set of all pre-restructuring DMUs except those belonging to $P$, where $P$ is the set of selected pre-restructuring DMUs in the above definition of a generalized restructuring and $\bar{P}=\{1, \ldots, n\}-P$. Assume that all the pre-restructuring DMUs belonging to $P$ disappear in the post-restructuring market. The proposed method in this paper can be easily modified to also cover other scenarios, say, when some of the pre-restructuring DMUs in $P$ are still available in the post-restructuring market. The generalized input-oriented InvDEA model is as follows. 


$$
\begin{array}{ll}
\min & \sum_{i \in I} \sum_{q \in Q} \sum_{p \in P} \alpha_{i p}^{q} \\
\text { s.t. } & \sum_{j \in \bar{P}} \lambda_{j}^{q} x_{i j}+\sum_{q \in Q} \sum_{p \in P} \alpha_{i p}^{q} \lambda_{q}-\bar{\theta}_{q} \sum_{p \in P} \alpha_{i p}^{q} \leq 0, i \in I, q \in Q \\
& \sum_{j \in \bar{P}} \lambda_{j}^{q} y_{r j}+\sum_{q \in Q} \sum_{p \in P} \beta_{r p}^{q} \lambda_{q}-\sum_{p \in P} \beta_{r p}^{q} \geq 0, \quad r \in R, q \in Q \\
& \sum_{j \in \bar{P}} \lambda_{j}^{q}+\lambda_{q}=1, q \in Q \\
& \sum_{q \in Q} \alpha_{i p}^{q} \leq x_{i p} \quad i \in I, p \in P \\
& \sum_{q \in Q} \beta_{r p}^{q}=y_{r p} \quad r \in R, p \in P \\
& \lambda_{j}^{q} \geq 0, j \in \bar{P}, q \in Q \\
& \lambda_{q} \geq 0, q \in Q \\
& \alpha_{i p}^{q} \geq 0, i \in I, q \in Q, p \in P \\
& \beta_{r p}^{q} \geq 0, r \in R, q \in Q, p \in P
\end{array}
$$

where, $\alpha_{i p}^{q}$ and $\beta_{r p}^{q}$ are respectively the levels of the $i^{\text {th }}$ input and the $r^{\text {th }}$ output that the post-restructuring $\mathrm{DMU}_{q}$ inherited from the pre-restructuring $\mathrm{DMU}_{p}$, for all $i \in I, r \in R, q \in Q, p \in P$, and $\bar{\theta}_{q}$ is the predefined efficiency target for the postrestructuring $\mathrm{DMU}_{q}$, for all $q \in Q$. The objective of the GInvDEA model (2) is to minimize the sum of inherited inputs, for each input of all pre-restructuring DMUs belonging to $P$, of all the post-restructuring DMUs belonging to $Q$ required to achieve their predefined efficiency targets. That is, the solution of the model suggests the maximum possible and required reduction in inputs of DMUs in $P$. It is worth noting that an InvDEA model deals with the determination of a vector of unknown parameters which would be a multiobjective programming. However, the main objective in a merger and/or acquisition is to determine the maximum achievable merger gains. The merger gain is the amount of inputs that can be saved by the merged entity and it is simply the sum of the saved inputs. This is the objective used in the proposed InvDEA model in Gattoufi et al. (2014) and in the current paper for the generalized restructuring. Nevertheless, if the decision maker seeks different objectives from a generalized restructuring there would be no restriction to consider multiobjective programming. 
Model (2) also assumes, as per its input-orientation, that there is no change in the aggregated outputs between the pre and post restructuring situations. Hence, there will be a redistribution of the assigned outputs between DMUs belonging to $Q$. Moreover, the model implicitly assumes that all inputs can be measured on the same scale. Nevertheless, alternative case can be seen from the utility theory angle, or using cost sharing approaches commonly discussed in economics and game theory. The following theorem shows that the NLP input-oriented GInvDEA model (2) can be linearized.

Theorem 1: The NLP model (2) can be simplified to the following LP model if and only if the corresponding pre and post restructuring efficiency frontiers are identical.

$$
\begin{aligned}
& \min \sum_{i \in I} \sum_{q \in Q} \sum_{p \in P} \alpha_{i p}^{q} \\
& \text { s.t. } \\
& \sum_{j \in \bar{P}} \lambda_{j}^{q} x_{i j}-\bar{\theta}_{q} \sum_{p \in P} \alpha_{i p}^{q} \leq 0, \quad i \in I, q \in Q \\
& \sum_{j \in \bar{P}} \lambda_{j}^{q} y_{r j}-\sum_{p \in P} \beta_{r p}^{q} \geq 0, \quad r \in R, q \in Q \\
& \sum_{j \in \bar{P}} \lambda_{j}^{q}=1, \quad q \in Q \\
& \sum_{q \in Q} \alpha_{i p}^{q} \leq x_{i p} \quad i \in I, p \in P \\
& \sum_{q \in Q} \beta_{r p}^{q}=y_{r p} \quad r \in R, p \in P \\
& \lambda_{j}^{q} \geq 0, j \in \bar{P}, q \in Q \\
& \alpha_{i p}^{q} \geq 0, i \in I, q \in Q, p \in P \\
& \beta_{r p}^{q} \geq 0, r \in R, q \in Q, p \in P
\end{aligned}
$$

Proof: Assume that the pre and post restructuring efficiency frontiers are identical. This means that the restructuring did not alter the pre-restructuring efficiency frontier. Mathematically, this means that all produced post-restructuring DMUs can be presented as a convex combination of some $\mathrm{DMU}_{j}$ for $j \in \bar{P}$. Therefore, in each optimal solution of model (2) we have $\lambda_{q}^{*}=0$ for all $q \in Q$. This completes the proof 
of sufficient condition. The necessary condition is straightforward and therefore this completes the proof

According to Theorem 1, the input-oriented GInvDEA model (3) can be used for determining the levels of inputs and outputs of $\bar{q}$ post-restructuring DMUs in $Q$ produced from $\bar{p}$ pre-restructuring DMUs in $P$. The objective of the GInvDEA model (3) guarantees that the aggregate unified value of inputs inherited by the post-restructuring DMUs from their pre-restructuring counterparts is minimized, in order to realize the desired efficiency targets $\bar{\theta}_{q}$ for all $q \in Q$.

The adoption of an input-oriented GInvDEA is recommended for a competitive market where the quantity of each output is controlled by the environment through the supply and demand equilibrium mechanism rather than by the firms themselves. In order to improve their performance, which is assumed to be the main objective of the restructuring, firms reduce the inputs' usage required to produce the inherited level of outputs to control costs and hence improve their profitability. Alternatively, in a not-for-profit environment where a public service is to be provided under tight budgetary restrictions, say, health care, performance improvement can be cast as a question of balancing the budget without reducing the overall service levels.

On the other hand, the adoption of an output-oriented GInvDEA is advised in a competitive market characterized by a demand largely exceeding the supply with limited resources available for the firms. In such situations, firms are willing to fully and wisely consume all available resources to maximize the incremental quantity produced of each output. Once again, an extension of output-orientation to the notfor-profit sector can be envisioned in the context of public policies designed to maximize service levels without concern for cutting back on existing expenditures. The output-oriented version of the GInvDEA is as follows. 


$$
\begin{aligned}
\max & \sum_{q \in Q} \sum_{r \in R} \beta_{r}^{q} \\
\text { s.t. } & \sum_{j \in \bar{P}} \lambda_{j}^{q} x_{i j}+\sum_{q \in Q} \sum_{p \in P} \alpha_{i p}^{q} \lambda_{q}-\sum_{p \in P} \alpha_{i p}^{q} \leq 0, i \in I, q \in Q \\
& \sum_{j \in \bar{P}} \lambda_{j}^{q} y_{r j}+\sum_{q \in Q} \sum_{p \in P} \beta_{r p}^{q} \lambda_{q}-\left(\sum_{p \in P} \beta_{r p}^{q}-\sum_{q \in Q} \beta_{r}^{q}\right) \bar{h}_{q} \geq 0, \quad r \in R, q \in Q \\
& \sum_{j \in \bar{P}} \lambda_{j}^{q}+\lambda_{q}=1, q \in Q \\
& \sum_{q \in Q} \alpha_{i p}^{q}=x_{i p} \quad i \in I, p \in P \\
& \sum_{q \in Q}\left(\beta_{r p}^{q}+\beta_{r}^{q}\right) \geq y_{r p} \quad r \in R, p \in P \\
& \lambda_{j}^{q} \geq 0, j \in \bar{P}, q \in Q \\
& \lambda_{q} \geq 0, q \in Q \\
& \alpha_{i p}^{q} \geq 0, i \in I, q \in Q, p \in P \\
& \beta_{r p}^{q} \geq 0, r \in R, q \in Q, p \in P \\
& \beta_{r}^{q} \geq 0, r \in R, q \in Q
\end{aligned}
$$

where, $\beta_{r}^{q}$ is the amount of the $r^{\text {th }}$ output that will be incrementally produced by the post-restructuring $\mathrm{DMU}_{q}$, for all $r \in R, q \in Q$, and $\bar{h}_{q}$ is the efficiency target for the post-restructuring $\mathrm{DMU}_{q}$ for all $q \in Q$.

Theorem 2 addresses the linearization of the output-oriented GInvDEA model (4).

Theorem 2: The NLP model (4) can be simplified to the following LP model if and only if the corresponding pre and post restructuring efficiency frontiers are identical. 


$$
\begin{aligned}
& \max \sum_{q \in Q} \sum_{r \in R} \beta_{r}^{q} \\
& \text { s.t. } \\
& \qquad \sum_{j \in \bar{P}} \lambda_{j}^{q} x_{i j}-\sum_{p \in P} \alpha_{i p}^{q} \leq 0, \quad i \in I, q \in Q \\
& \sum_{j \in \bar{P}} \lambda_{j}^{q} y_{r j}-\left(\sum_{p \in P} \beta_{r p}^{q}-\sum_{q \in Q} \beta_{r}^{q}\right) \bar{h}_{q} \geq 0, \quad r \in R, q \in Q \\
& \sum_{j \in \bar{P}} \lambda_{j}^{q}=1, q \in Q \\
& \sum_{q \in Q} \alpha_{i p}^{q}=x_{i p} \quad i \in I, p \in P \\
& \sum_{q \in Q}\left(\beta_{r p}^{q}+\beta_{r}^{q}\right) \geq y_{r p} \quad r \in R, p \in P \\
& \lambda_{j}^{q} \geq 0, j \in \bar{P}, q \in Q \\
& \alpha_{i p}^{q} \geq 0, i \in I, q \in Q, p \in P \\
& \beta_{r p}^{q} \geq 0, r \in R, q \in Q, p \in P \\
& \beta_{r}^{q} \geq 0, r \in R, q \in Q
\end{aligned}
$$

Proof: It is straightforward.

In the following, we show that the generalized restructuring InvDEA model (2) can be linearized without any restriction on the post-restructuring frontier. Assume a post-restructuring generated DMU- $q(q \in Q)$ falls outside the pre-restructuring frontier. In this case, there is an optimal solution such that $\lambda_{q}=1$. Therefore, in model (2) we can assume that $\lambda_{q} \in\{0,1\}$ for all $q \in Q$. This implies that the nonlinear terms of model (2) can be linearized using the following substitutions.

$$
\begin{array}{ll}
\alpha_{i p}^{q} \lambda_{q}=\hat{\alpha}_{i p}^{q} & i \in I, p \in P, q \in Q \\
\beta_{r p}^{q} \lambda_{q}=\hat{\beta}_{i p}^{q} & r \in R, p \in P, q \in Q
\end{array}
$$

Where,

$$
\begin{array}{ll}
\hat{\alpha}_{i p}^{q} \leq x_{i p} \alpha_{i p}^{q} & i \in I, p \in P, q \in Q \\
\alpha_{i p}^{q}-\left(1-\lambda_{q}\right) x_{i p} \leq \hat{\alpha}_{i p}^{q} \leq \alpha_{i p}^{q} & i \in I, p \in P, q \in Q
\end{array}
$$

and 


$$
\begin{aligned}
& \hat{\beta}_{r p}^{q} \leq y_{r p} \beta_{r p}^{q} \quad r \in R, p \in P, q \in Q \\
& \beta_{r p}^{q}-\left(1-\lambda_{q}\right) y_{r p} \leq \hat{\beta}_{r p}^{q} \leq \beta_{r p}^{q} \quad r \in R, p \in P, q \in Q
\end{aligned}
$$

Therefore, the nonlinear model (2) can be linearized to the following model

$$
\begin{array}{ll}
\min & \sum_{i \in I} \sum_{q \in Q} \sum_{p \in P} \alpha_{i p}^{q} \\
\text { s.t. } & \sum_{j \in \bar{P}} \lambda_{j}^{q} x_{i j}+\sum_{q \in Q} \sum_{p \in P} \hat{\alpha}_{i p}^{q}-\bar{\theta}_{q} \sum_{p \in P} \alpha_{i p}^{q} \leq 0, i \in I, q \in Q \\
& \sum_{j \in \bar{P}} \lambda_{j}^{q} y_{r j}+\sum_{q \in Q} \sum_{p \in P} \hat{\beta}_{r p}^{q}-\sum_{p \in P} \beta_{r p}^{q} \geq 0, \quad r \in R, q \in Q \\
& \sum_{j \in P} \lambda_{j}^{q}+\lambda_{q}=1, q \in Q \\
& \sum_{q \in Q} \alpha_{i p}^{q} \leq x_{i p} \quad i \in I, p \in P \\
& \sum_{q \in Q} \beta_{r p}^{q}=y_{r p} \quad r \in R, p \in P \\
& \hat{\alpha}_{i p}^{q} \leq x_{i p} \alpha_{i p}^{q} \\
& \alpha_{i p}^{q}-\left(1-\lambda_{q}\right) x_{i p} \leq \hat{\alpha}_{i p}^{q} \leq \alpha_{i p}^{q} \quad i \in I, p \in P, q \in Q \\
& \hat{\beta}_{r p}^{q} \leq y_{r p} \beta_{r p}^{q} \\
\beta_{r p}^{q}-\left(1-\lambda_{q}\right) y_{r p} \leq \hat{\beta}_{r p}^{q} \leq \beta_{r p}^{q} \quad r \in R, p \in P, q \in Q \\
\lambda_{j}^{q} \geq 0, j \in \bar{P}, q \in Q \\
\lambda_{q} \in\{0,1\}, q \in Q \\
\alpha_{i p}^{q} \geq 0, \hat{\alpha}_{i p}^{q} \geq 0, i \in I, q \in Q, p \in P \\
\beta_{r p}^{q} \geq 0, \hat{\beta}_{r p}^{q} \geq 0, r \in R, q \in Q, p \in P
\end{array}
$$

As shown above, model $(\overline{2})$ does not need any restriction on post-restructuring frontier and therefore can be used if model (3) is infeasible. This would be a generalized restructuring with frontier change if authorities and policy makers allow such a change in the market.

An important advantage of the proposed GInvDEA models (3) and (5), compared to the InvDEA models suggested in Gattoufi et al. (2014), resides in the fact that both models (3) and (5) define all $\bar{q}$ post-restructuring DMUs simultaneously by solving only one model, not iteratively by using InvDEA model. The following figure illustrates the fact that the InvDEA method is not appropriate for a generalized 
restructuring case. Figure 1 shows a numerical example with five DMUs, A to F, each with one input and one output. Consider a generalized restructuring where three pre-restructuring DMUs D, E, and F, decide to consolidate their activities by generating two post-restructuring DMUs, i. e. $\bar{p}=3, \bar{q}=2$.

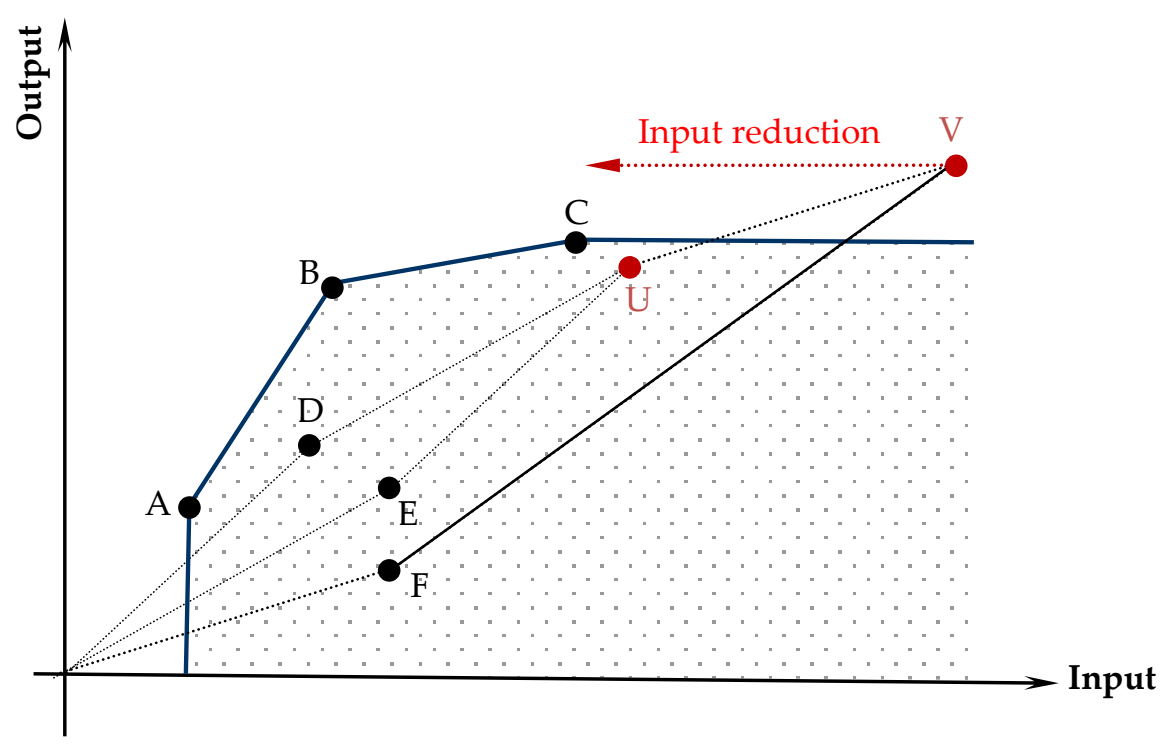

Figure 1: Illustration for InvDEA

If model (1) is adopted, it leads to the need of splitting the virtual DMU V. The latter is generated by the sequence of mergers between $\mathrm{D}$ and $\mathrm{E}$ first to produce the virtual DMU U, then between $\mathrm{U}$ and $\mathrm{F}$ to produce DMU V as shown in Figure 1. Ultimately, there will be a need to split the virtual DMU V into two post-restructuring DMUs, which is beyond the capability of the InvDEA method.

Alternatively, the GInvDEA method defines the post-restructuring DMUs by reallocating and reassigning the inherited inputs and outputs simultaneously. Assume, for illustration, that two post-restructuring DMUs are targeting to be fully efficient, or $\bar{\theta}_{1}=\bar{\theta}_{2}=1$. Solving the proposed GInvDEA model (3) defines the postrestructuring set of DMUs. The illustration is shown in Figure 2.

Any couple of fully efficient DMUs with aggregate input and output equal to those of the virtual DMU V is a solution. The illustration shows that the input of the virtual DMU V is reduced first, keeping the output at the same level, to create $V_{1}$. 
The solution $\left(C_{1}, C_{2}\right)$ is generated by reallocating the input and output of $V_{1}$ as shown in Figure 2.

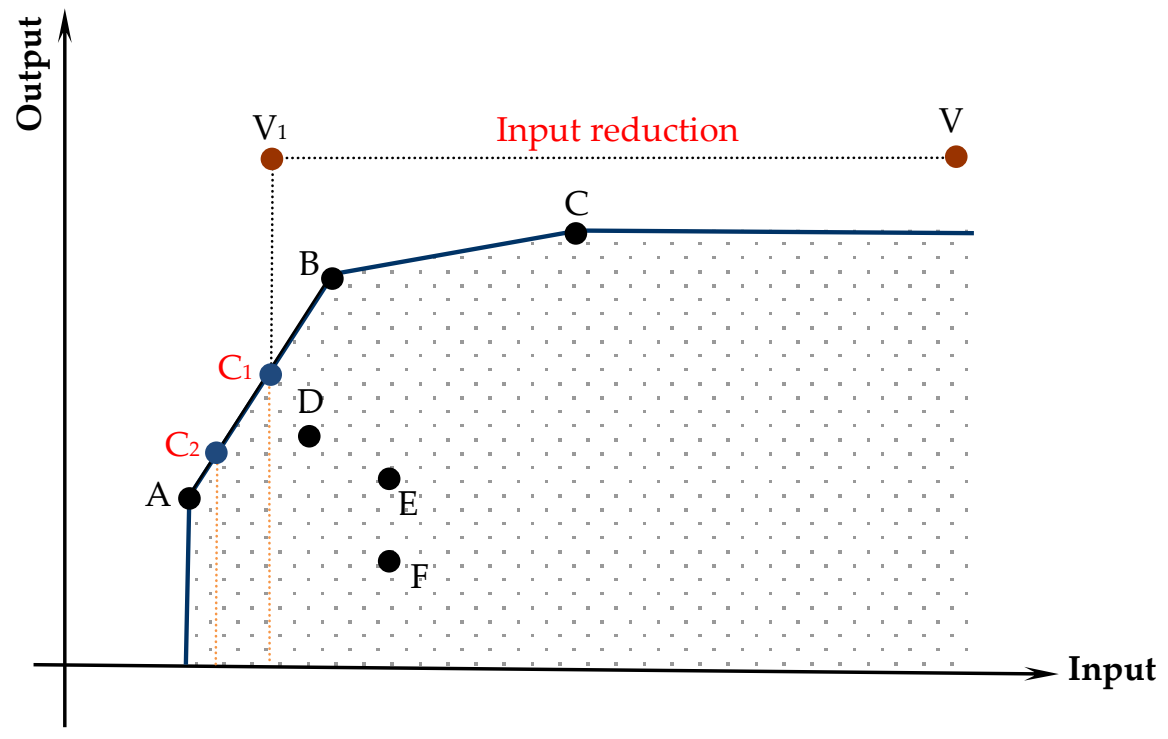

Figure 2: Illustration for GInvDEA

It would be institutive to note that model (1) cannot be used for a generalized restructuring situation. The proposed GInvDEA model creates $q$ post-restructuring DMUs by redistributing the inputs and outputs of $p$ pre-restructuring DMUs. These decision variables, the inputs and outputs of the post-restructuring DMUs, are related to each other and cannot be obtained separately. Assume we start distributing the inherited inputs and outputs of the pre-restructuring DMUs to create the first post-restructuring DMU in the input orientation. In this case, the corresponding model allocates minimum level of inputs, according to the objective function, and as much outputs as possible to realize the given efficiency target for the first post-restructuring DMU. There is no guarantee to be able to create all postrestructuring DMUs using this reallocation methodology as it distributes the minimum inputs and as much outputs as required to realize the efficiency targets.

\section{Minimum achievable efficiency targets}

The feasibility of the proposed GInvDEA models (3) and (5) depends on the efficiency targets of the post-restructuring DMUs. Knowing the minimum achievable 
efficiency targets has a high informative value for the decision-maker deliberating about engaging in the restructuring process. This information sheds light on the lowest efficiency score that can be realized by the post-restructuring DMUs.

The proof of the following theorem is omitted.

Theorem 3: If model (3) is feasible for the efficiency targets $\bar{\theta}_{q}$, for all $q \in Q$, then it remains feasible for all efficiency targets $\hat{\theta}_{q}$, where $\bar{\theta}_{q} \leq \hat{\theta}_{q} \leq 1$ for all $q \in Q$.

A legitimate inquiry by any decision maker involved in a restructuring program would be to know the lowest efficiency score that can be realized by each postrestructuring DMU. The following linear programming model addresses this concern.

$$
\begin{aligned}
& \min \sum_{q \in Q} \theta_{q} \\
& \text { s.t. } \\
& \sum_{j \in \bar{P}} \lambda_{j}^{q} x_{i j}-\sum_{p \in P} \alpha_{i p}^{q} \leq 0, \quad i \in I, q \in Q \\
& \sum_{j \in \bar{P}} \lambda_{j}^{q} y_{r j}-\sum_{p \in P} \beta_{r p}^{q} \geq 0, \quad r \in R, q \in Q \\
& \sum_{j \in \bar{P}} \lambda_{j}^{q}=1, \quad q \in Q \\
& \sum_{q \in Q} \alpha_{i p}^{q} \leq x_{i p} \quad i \in I, p \in P \\
& \sum_{q \in Q} \beta_{r p}^{q}=y_{r p} \quad r \in R, p \in P \\
& \sum_{p \in P} \alpha_{i p}^{q}-\theta_{q} \sum_{p \in P} x_{i p} \leq 0 \quad i \in I, q \in Q \\
& \lambda_{j}^{q} \geq 0, j \in \bar{P}, q \in Q \\
& \alpha_{i p}^{q} \geq 0, i \in I, q \in Q, p \in P \\
& \beta_{r p}^{q} \geq 0, r \in R, q \in Q, p \in P \\
& \theta_{q} \geq 0, q \in Q
\end{aligned}
$$

The objective in model (6) is to minimize the sum of the efficiency scores that can be realized by the post-restructuring DMUs. The first set of constraints, by ignoring the efficiency targets, is similar to the set of constraints in model (3). As per classical DEA model, the last set of constraints guarantees that the sum of the allocated inherited inputs by each post-restructuring DMU is at most equal to the product of 
the minimum realized efficiency score by the aggregated inputs of the prerestructuring DMUs.

Solving this model has a critical importance for the restructuring' decision maker. The solution is very informative since it provides foresight about the postrestructuring positioning of the restructured units. It provides the lowest bound of performance each post-restructuring unit can realize. Hence, the decision of restructuring can be made if those lowest bounds are judged satisfactory.

Theorem 4: The lowest efficiency scores realized through a generalized restructuring can be determined by solving the corresponding model (6).

Proof: Consider a generalized restructuring with $\bar{\theta}_{q}, q \in Q$, as the efficiency targets for all post-restructuring DMUs. It is sufficient to show that $\bar{\theta}_{q} \geq \theta_{q}^{*}$ for all $q \in Q$, where $\theta_{q}^{*}$ is taken from an optimal solution of the corresponding model (6). Assume on the contrary that $\bar{\theta}_{q}<\theta_{q}^{*}$ for some $q \in Q$. Clearly, any feasible solution of model (3), corresponding to $\bar{\theta}_{q}, q \in Q$, can be converted to a feasible solution in model (6). This simply implies that the optimal value of model (6) can be further improved. This is a contradiction and therefore it completes the proof.

An ideal generalized restructuring would be a scenario where the corresponding optimal value of model (6) is equal to $\sum_{q \in Q} \theta_{q}^{*}=\bar{q}$, which imposes that all postrestructuring DMUs are fully efficient. This situation represents a process that produces at worst fully efficient post-restructuring DMUs, hence strongly recommended. It is an exceptional opportunity. 


\section{An application in banking operations}

This section provides an application of the proposed GInvDEA method in banking. Nevertheless, any other sector with homogeneous firms having the possibility of restructuring can be a subject for the GInvDEA method. The data used in this section, reported in Appendix 1 and reproduced from Gattoufi et al (2014), lists the inputs and outputs values for 42 commercial GCC banks assumed to be using two inputs to produce two outputs. The GCC commercial banks financial data is obtained from BANKSCOPE database for the year 2010.

In line with the intermediation theory for banking, as discussed in Berger and Humphrey (1997) and Avkiran (2009), the two inputs considered are interest expenses and non-interest expenses while the two outputs are interest income and non-interest income.

For the illustration, we consider the two types of the generalized restructuring defined earlier in this paper. The first case is about a set of three pre-restructuring banks that produce two post-restructuring banks through consolidation. For the split case, two pre-restructuring banks are assumed to split into a set of three postrestructuring ones.

Assume that banks $\mathrm{B}_{01}, \mathrm{~B}_{02}$, and $\mathrm{B}_{03}$ are considering consolidating their activities by generating two new banks. Therefore, the sets of pre and post restructuring indices will be $\mathrm{P}=\{1,2,3\}, \mathrm{Q}=\{1,2\}$ respectively and $\overline{\mathrm{P}}=\{4, \ldots, 42\}$.

The corresponding inputs and outputs for the post-restructuring banks, denoted $\mathrm{C}_{1}$ and $\mathrm{C}_{2}$, are

$$
\mathrm{C}_{1}=\left(\begin{array}{l}
\alpha_{11}^{1}+\alpha_{12}^{1}+\alpha_{13}^{1} \\
\alpha_{21}^{1}+\alpha_{22}^{1}+\alpha_{23}^{1} \\
\beta_{11}^{1}+\beta_{12}^{1}+\beta_{13}^{1} \\
\beta_{11}^{1}+\beta_{12}^{1}+\beta_{13}^{1}
\end{array}\right) \& \mathrm{C}_{2}=\left(\begin{array}{l}
\alpha_{11}^{2}+\alpha_{12}^{2}+\alpha_{13}^{2} \\
\alpha_{21}^{2}+\alpha_{22}^{2}+\alpha_{23}^{2} \\
\beta_{11}^{2}+\beta_{12}^{2}+\beta_{13}^{2} \\
\beta_{11}^{2}+\beta_{12}^{2}+\beta_{13}^{2}
\end{array}\right)
$$

and the corresponding input-oriented GInvDEA (3) is 
$\operatorname{Min}\left(\alpha_{11}^{1}+\alpha_{12}^{1}+\alpha_{13}^{1}\right)+\left(\alpha_{11}^{2}+\alpha_{12}^{2}+\alpha_{13}^{2}\right)+\left(\alpha_{21}^{1}+\alpha_{22}^{1}+\alpha_{23}^{1}\right)+\left(\alpha_{21}^{2}+\alpha_{22}^{2}+\alpha_{23}^{2}\right)$

s.t.

$$
\begin{aligned}
& \sum_{j \in \bar{P}} \lambda_{j}^{1} x_{i j}-\left(\alpha_{i 1}^{1}+\alpha_{i 2}^{1}+\alpha_{i 3}^{1}\right) \times \bar{\theta}_{1} \leq 0 i=1,2 \\
& \sum_{j \in \bar{P}} \lambda_{j}^{2} x_{i j}-\left(\alpha_{i 1}^{2}+\alpha_{i 2}^{2}+\alpha_{i 3}^{2}\right) \times \bar{\theta}_{2} \leq 0 i=1,2 \\
& \sum_{j \in \bar{P}} \lambda_{j}^{1}=1 \\
& \sum_{j \in \bar{P}} \lambda_{j}^{2}=1 \\
& \sum_{j \in \bar{P}} \lambda_{j}^{1} y_{r j}-\left(\beta_{r 1}^{1}+\beta_{r 2}^{1}+\beta_{r 3}^{1}\right) \geq 0 \quad r=1,2 \\
& \sum_{j \in \bar{P}} \lambda_{j}^{2} y_{r j}-\left(\beta_{r 1}^{2}+\beta_{r 2}^{2}+\beta_{r 3}^{2}\right) \geq 0 \quad r=1,2 \\
& \alpha_{11}^{1}+\alpha_{11}^{2} \leq 3956.796, \alpha_{12}^{1}+\alpha_{12}^{2} \leq 481.239 \\
& \alpha_{13}^{1}+\alpha_{13}^{2} \leq 305.2, \alpha_{21}^{1}+\alpha_{21}^{2} \leq 1894.426 \\
& \alpha_{22}^{1}+\alpha_{22}^{2} \leq 319.976, \alpha_{23}^{1}+\alpha_{23}^{2} \leq 138.6 \\
& \beta_{11}^{1}+\beta_{11}^{2}=9001.004, \beta_{12}^{1}+\beta_{12}^{2}=974.854 \\
& \beta_{13}^{1}+\beta_{13}^{2}=479.8, \beta_{21}^{1}+\beta_{21}^{2}=870.497 \\
& \beta_{22}^{1}+\beta_{22}^{2}=597.726, \beta_{23}^{1}+\beta_{23}^{2}=252.2 \\
& \alpha_{i p}^{q} \geq 0, \beta_{r p}^{q} \geq 0, \lambda_{j}^{q} \geq 0, q \in Q, p \in P, j \in \bar{P}
\end{aligned}
$$

The minimum efficiency scores, determined by solving model (6), that the two postrestructuring banks $C_{1}$ and $C_{2}$ can realize are respectively $\theta_{1}^{*}=0.5$ and $\theta_{2}^{*}=0.4831$. Based on Theorem 4, if the two conditions $\bar{\theta}_{1} \geq 0.5$ and $\bar{\theta}_{2} \geq 0.4831$ are violated, the corresponding GInvDEA model (3) will be infeasible. If these "pessimistic" levels are judged satisfactory, the decision maker is encouraged to engage the restructuring. Assuming that the new banks $C 1$ and $C_{2}$ target to be fully efficient, that is $\bar{\theta}_{1}=\bar{\theta}_{2}=1$ , the above GInvDEA model has the following optimal solution.

$$
\begin{aligned}
& \lambda_{10}^{1}=0.3795, \lambda_{20}^{1}=0.1423, \lambda_{24}^{1}=0.2419, \lambda_{36}^{1}=0.2363 ; \text { and } \lambda_{j}^{1}=0 \text { for } j \notin\{10,20,24,36\} \\
& \lambda_{10}^{2}=0.9973, \text { and } \lambda_{36}^{2}=0.0027 ; \text { and } \lambda_{j}^{2}=0 \text { for } j \notin\{10,36\} \\
& \alpha_{11}^{1}=3574.51, \alpha_{12}^{1}=329.7038, \alpha_{13}^{1}=305.2 \text { and } \alpha_{11}^{2}=382.285, \alpha_{12}^{2}=0, \alpha_{13}^{2}=0 \\
& \alpha_{21}^{1}=1635.21, \alpha_{22}^{1}=319.976, \alpha_{23}^{1}=138.6 \text { and } \alpha_{21}^{2}=259.2192, \alpha_{22}^{2}=0, \alpha_{23}^{2}=0 \\
& \beta_{11}^{1}=9001, \beta_{12}^{1}=0, \beta_{13}^{1}=479.8, \text { and } \beta_{11}^{2}=0, \beta_{12}^{2}=974.854, \beta_{13}^{2}=0 \\
& \beta_{21}^{1}=7571.33, \beta_{22}^{1}=0, \beta_{23}^{1}=0, \text { and } \beta_{21}^{2}=1130.16, \beta_{22}^{2}=597.726, \beta_{23}^{2}=252.2
\end{aligned}
$$


Therefore,

$$
C_{1}=\left(\begin{array}{l}
4209.412 \\
2093.780 \\
9480.800 \\
7571.330
\end{array}\right) \quad \mathcal{E} C_{2}=\left(\begin{array}{l}
382.285 \\
259.219 \\
974.854 \\
1980.090
\end{array}\right)
$$

The case of a split constitutes the second illustrative example. Assume that the two banks $\mathrm{B}_{08}$ and $\mathrm{B}_{12}$ consider restructuring their activities through a split into three post-restructuring banks. Therefore, the sets of pre and post restructuring banks' indices are $P=\{8,12\}, Q=\{1,2,3\}$ respectively and $\bar{P}=\{1, \ldots, 42\} \backslash\{8,12\}$. Let $S_{1}, S_{2}$ and $S_{3}$ be the three banks generated by the split. Therefore,

$$
\mathrm{S}_{q}=\left(\begin{array}{l}
\alpha_{11}^{q}+\alpha_{12}^{q} \\
\alpha_{21}^{q}+\alpha_{22}^{q} \\
\beta_{11}^{q}+\beta_{12}^{q} \\
\beta_{11}^{q}+\beta_{12}^{q}
\end{array}\right), \quad q=1,2,3
$$

The corresponding input-oriented GInvDEA model (3) becomes

$$
\begin{aligned}
& \text { Min }\left(\alpha_{11}^{1}+\alpha_{12}^{1}\right)+\left(\alpha_{11}^{2}+\alpha_{12}^{2}\right)+\left(\alpha_{11}^{3}+\alpha_{12}^{3}\right)+\left(\alpha_{21}^{1}+\alpha_{22}^{1}\right)+\left(\alpha_{21}^{2}+\alpha_{22}^{2}\right)+\left(\alpha_{21}^{3}+\alpha_{22}^{3}\right) \\
& \text { s.t. } \\
& \quad \sum_{j \in \bar{P}} \lambda_{j}^{q} x_{i j}-\left(\alpha_{i 1}^{q}+\alpha_{i 2}^{q}\right) \times \bar{\theta}_{q} \leq 0 \quad i=1,2, q=1,2,3 \\
& \sum_{j \in \bar{P}} \lambda_{j}^{q}=1 \quad q=1,2,3 \\
& \sum_{j \in \bar{P}} \lambda_{j}^{q} y_{r j}-\left(\beta_{r 1}^{q}+\beta_{r 2}^{q}\right) \geq 0 \quad r=1,2, q=1,2,3 \\
& \alpha_{11}^{1}+\alpha_{11}^{2}+\alpha_{11}^{3} \leq 14.63, \alpha_{12}^{1}+\alpha_{12}^{2}+\alpha_{12}^{3} \leq 14.665 \\
& \alpha_{21}^{1}+\alpha_{21}^{2}+\alpha_{21}^{3} \leq 16.863, \alpha_{22}^{1}+\alpha_{22}^{2}+\alpha_{22}^{3} \leq 8.973 \\
& \beta_{11}^{1}+\beta_{11}^{2}+\beta_{11}^{3}=44.659, \beta_{12}^{1}+\beta_{12}^{2}+\beta_{12}^{3}=28.124 \\
& \beta_{21}^{1}+\beta_{21}^{2}+\beta_{21}^{3}=14.938, \beta_{22}^{1}+\beta_{22}^{2}+\beta_{22}^{3}=10.971 \\
& \lambda_{j}^{q} \geq 0, \forall j \in \bar{P}, q=1,2,3 \\
& \alpha_{i p}^{q} \geq 0, i=1,2, p=1,2, q=1,2,3 \\
& \beta_{r p}^{q} \geq 0, r=1,2, p=1,2, q=1,2,3
\end{aligned}
$$


The minimum achievable efficiency scores for the three post-restructuring banks are, respectively, $\theta_{1}^{*}=0.2967, \theta_{2}^{*}=0.2027$, and $\theta_{3}^{*}=0.2244$. Based on Theorem 4 , if the three conditions $\bar{\theta}_{1} \geq 0.2967, \bar{\theta}_{2} \geq 0.2027$, and $\bar{\theta}_{3} \geq 0.2244$ are violated, or equivalently $\sum_{q \in Q} \bar{\theta}_{q}<\sum_{q \in Q} \theta_{q}^{*}$, the corresponding GInvDEA model (3) will be infeasible. According to Theorem 3, the GInvDEA model (3) is feasible for all efficiency targets $\bar{\theta}_{1}, \bar{\theta}_{2}$ and $\bar{\theta}_{3}$ that satisfy $0.2967 \leq \bar{\theta}_{1} \leq 1,0.2027 \leq \bar{\theta}_{2} \leq 1$, and $0.2244 \leq \bar{\theta}_{3} \leq 1$.

For the special case where the split banks $S_{q}(q=1,2,3)$ are to be fully efficient, $\bar{\theta}_{q}=1, q=1,2,3$, an optimal solution of the above model is

$$
\begin{aligned}
& \lambda_{5}^{1}=0.9668, \lambda_{31}^{1}=0.0332, \lambda_{5}^{2}=0.9576, \lambda_{31}^{2}=0.0424, \lambda_{5}^{3}=0.9824, \lambda_{31}^{3}=0.0176 \\
& \lambda_{j}^{q}=0, \forall j \in \bar{P}, j \neq 5,31, q=1,2,3 \\
& \alpha_{12}^{1}=6.0444, \alpha_{21}^{1}=7.5555, \alpha_{11}^{2}=7.4481, \alpha_{21}^{2}=9.3075, \alpha_{11}^{3}=3.6869, \alpha_{22}^{3}=4.6131 \\
& \beta_{11}^{1}=25.6928, \beta_{21}^{1}=14.938, \beta_{22}^{1}=10.971, \beta_{11}^{2}=3.8913, \beta_{12}^{2}=28.124, \beta_{11}^{3}=15.0749
\end{aligned}
$$

Therefore, we have the following table

Table 1: Distributed inherited inputs and outputs between three split banks

\begin{tabular}{ccccc}
\hline $\begin{array}{c}\text { Generated } \\
\text { banks }\end{array}$ & $\begin{array}{c}\text { Interest } \\
\text { expenses }\end{array}$ & $\begin{array}{c}\text { Non-interest } \\
\text { expenses }\end{array}$ & $\begin{array}{c}\text { Interest } \\
\text { incomes }\end{array}$ & $\begin{array}{c}\text { Non-interest } \\
\text { incomes }\end{array}$ \\
\hline $\mathrm{S}_{1}$ & 6.0444 & 7.5555 & 25.6928 & 25.909 \\
$\mathrm{~S}_{2}$ & 7.4481 & 9.3075 & 32.0153 & 0.000 \\
$\mathrm{~S}_{3}$ & 3.6869 & 4.6131 & 15.0749 & 0.000 \\
\hline
\end{tabular}

The solution shown in Table 1 recommends a zero-value non-interest income for the two split banks $S_{2}$ and $S_{3}$. Hence, the two mentioned banks are advised to revise their product mix by concentrating their activity solely in products generating interest income, in order to realize full efficiency.

\section{Concluding remarks}

This paper introduces the new concept of generalized firms restructuring. It is defined as a move in a business market when a given group of at least two pre- 
restructuring decision making units (DMUs) decide to go through either a consolidation resulting in at most the same number of post-restructuring DMUs, or through a split that generates at least the same number of post-restructuring DMUs. While the consolidation is intended to realize synergies, the split is usually a means for reverse synergies.

This paper genuinely extends and links the state of the art in the areas of firm restructuring and Data Envelopment Analysis (DEA). It enriches the firm restructuring literature by introducing new concepts and developing new analytical tools for decision making. A novel method for modelling a generalized restructuring using inverse DEA, called GInvDEA, is suggested in this paper. The solution of the proposed model advises about optimal sharing by the post-restructuring DMUs of the inherited inputs and outputs from the pre-restructuring ones, to realize their desired efficiency targets. The paper also suggests an insightful method for finding the lowest efficiency scores post-restructuring DMUs can achieve. If satisfactory, this minimum can be considered as a threshold for the adoption of the corresponding generalized restructure.

Further generalization of the current research consists of considering the case of a generalized restructuring scenario with an unknown number of pre and post restructuring DMUs.

\section{References}

Avkiran, N.C., (1999). The evidence on efficiency gains: The role of mergers and the benefits to the public. Journal of Banking \& Finance, 23(7), 991-1013.

Avkiran, N.C., (2009). Opening the black box of efficiency analysis: An illustration with UAE banks. Omega, 37(4), 930-941.

Berger, A.N., Humphrey, D.B., (1997). Efficiency of financial institutions: International survey and directions for future research. European Journal of Operational Research, 98(2), $175-212$. 
Bogetoft, P., Strange N., Thorsen, B.J., (2003). Efficiency and merger gains in the Danish forestry extension service. Forest Science, 49(4), 585-595.

Bogetoft, P., Wang, D.X., (2005). Estimating the potential gains from mergers. Journal of Productivity Analysis, 23(2), 145-171.

De Witte, K., Dijkgraaf, E., (2010). Mean and bold? On separating merger economies from structural efficiency gains in the drinking water sector. Journal of the Operational Research Society, 61(2), 222-234.

Ferrier, G.D., Valdmanis, V.G., (2004). Do mergers improve hospital productivity? Journal of the Operational Research Society, 55(10), 1071-1080.

Frija, A., Wossink, A., Buysse, J., Speelman, S., Van Huylenbroeck, G., (2011). Irrigation pricing policies and its impact on agricultural inputs demand in Tunisia: A DEA-based methodology. Journal of Environmental Management, 92(9), 2109-2118.

Gattoufi, S., Amin, G.R., Emrouznejad, A., (2014). A new inverse DEA method for merging banks. IMA Journal of Management Mathematics, 24(6), 78-98.

Gaughan, P.A., (2010). Mergers, Acquisitions, and corporate restructuring, $5^{\text {th }}$ edition, John Wiley, USA.

Ghiyasi, M. (2015) On inverse DEA model: The case of variable returns to scale. Computers $\mathcal{E}$ Industrial Engineering, 87, 407-409.

Ghobadi, S., Jahangiri, S., (2015). Inverse DEA: Review, Extension and Application. International Journal of Information Technology \& Decision Making, 14(4), 805-824.

Hadi-Vencheh, A., Foroughi, A.A., (2006). A generalized DEA model for inputs/outputs estimation. Mathematical and Computer Modelling, 43, 447-457.

Hadi-Vencheh, A., Foroughi, A. A., and Soleimani-damaneh, M. (2008). A DEA model for resource allocation. Economic Modelling, 25(5), 983-993.

Hadi-Vencheh, A., Hatami-Marbini, A., Ghelej Beigi, Z., Gholami, K., (2015). An inverse optimization model for imprecise data envelopment analysis. Optimization, 64(11), 24412454.

Halkos, G.E., Matousek, R., Tzeremes, N.G., (2015). Pre-evaluating technical efficiency gains from possible mergers and acquisitions: evidence from Japanese regional banks. Review of Quantitative Finance and Accounting, article in press, Doi: 10.1007/s11156-014-0461-5.

Halkos, G.E., Tzeremes, N.G., (2013). Estimating the degree of operating efficiency gains from a potential bank merger and acquisition: A DEA bootstrapped approach. Journal of Banking \& Finance, 37(5), 1658-1668.

Harris, J., Ozgen, H., Ozcan, Y., (2000). Do mergers enhance the performance of hospital efficiency? Journal of the Operational Research Society, 51(7), 801-811.

Jahanshahloo, G.R., Hadi-Vencheh, A., Foroughi, A.A., Kazemi Matin, R., (2004a). Inputs/outputs estimation in DEA when some factors are undesirable. Applied Mathematics and Computation, 156, 19-32. 
Jahanshahloo, G.R., Hosseinzadeh Lotfi, F., Shoja, N., Tohidi, G., Razavyan, S., (2005). Sensitivity of efficiency classifications in the inverse DEA models. Applied Mathematics and Computation, 169, 905-916.

Jahanshahloo, G. R., Hosseinzadeh Lotfi, F., Shoja, N., Tohidi, G., and Razavyan, S. (2004b). Input estimation and identification of extra in inverse DEA models. Applied Mathematics and Computation, 156, 427-437.

Jahanshahloo, G.R., Soleimani-damaneh, M., Ghobadi, S., (2015). Inverse DEA under intertemporal dependence using multiple-objective programming. European Journal of Operational Research, 240(2), 447-456.

Kong, C., Chow, W., Ka, M., Fung, Y., (2012). Measuring the effects of China's airline mergers on the productivity of state-owned carriers. Journal of Air Transport Management 25(1), 1-4.

Kristensen, T., Bogetoft, P., Pedersen, K.M., (2010). Potential gains from hospital mergers in Denmark. Health Care Management Science, 13(4), 334-345.

Kwoka, J., Pollitt, M., (2010). Do mergers improve efficiency? Evidence from restructuring the US electric power sector. International Journal of Industrial Organization, 28(6), 645-656.

Leleu, H., Moises, J., Valdmanis, V., (2012). Optimal productive size of hospital's intensive care units. International Journal of Production Economics, 136(2), 297-305.

Lertworasirikul, S. Charnsethikul, P. and Fang, S.C., (2011). Inverse data envelopment analysis model to preserve relative efficiency values: The case of variable returns to scale. Computers \& Industrial Engineering, 61(4), 1017-1023.

Lim, D.J., (2016). Inverse DEA with frontier changes for new product target setting. European Journal of Operational Research, 254(2), 510-516.

Lin, H.T., (2010). An efficiency-driven approach for setting revenue target. Decision Support Systems, 49, 311-317.

Liu, H.H., Chen, T.Y., Pai, L.Y., (2007). The influence of merger and acquisition activities on corporate performance in the Taiwanese telecommunications industry. The Service Industries Journal, 27(7-8), 1041-1051.

Lozano, S., Villa, G., (2010). DEA-based pre-merger planning tool. Journal of the Operational Research Society, 61(10), 1485-1497.

Peyrache, A., (2013). Industry structural inefficiency and potential gains from mergers and break-ups: A comprehensive approach. European Journal of Operational Research, 230(2), 422-430.

Wei, Q., Zhang, J., Zhang, X., (2000). An inverse DEA model for inputs/outputs estimate. European Journal of Operational Research, 121(1), 151-163.

Wu D, Luo C, Wang H, Birge JR (2014). Bi-level Programming Merger Evaluation and Application to Banking Operations. Production and Operations Management. Accepted and in press. DOI: 10.1111/poms.12205.

Wu, D.D., Birge, J.R., (2011). Serial chain merger evaluation model and application to mortgage banking. Decision Sciences, 43(1), 5-36. 
Wu, D.D., Zhou, Z., Birge, J.R., (2011). Estimation of potential gains from mergers in multiple periods: a comparison of stochastic frontier analysis and data envelopment analysis. Annals of Operations Research, 186(1), 357-381.

Yan, H., Wei, Q., and Hao, G., (2002). DEA models for resource reallocation and production input/output estimation. European Journal of Operational Research, 136(1), 19-31.

Zhang, X.S. and Cui, J.C., (1999). A project evaluation system in the state economic information system of china an operations research practice in public sectors. International Transactions in Operational Research, 6, 441-452.

Zhang, M., Cui, J.C., (2016). The extension and integration of the inverse DEA method. Journal of the Operational Research Society, in press, doi:10.1057/jors.2016.2. 
Appendix 1: GCC 42 banks data and efficiency scores

\begin{tabular}{|c|c|c|c|c|c|}
\hline Bank & $\begin{array}{l}\text { Interest } \\
\text { Expenses }\end{array}$ & $\begin{array}{l}\text { Non-Interest } \\
\text { expenses }\end{array}$ & $\begin{array}{l}\text { Interest } \\
\text { Incomes }\end{array}$ & $\begin{array}{c}\text { Non- } \\
\text { Interest } \\
\text { Incomes }\end{array}$ & $\begin{array}{l}\text { Technical Efficiency } \\
\text { scores under VRS }\end{array}$ \\
\hline B01 & 3956.796 & 1894.426 & 9001.004 & 8701.497 & 1.000 \\
\hline B02 & 481.239 & 319.976 & 974.854 & 597.726 & 0.677 \\
\hline B03 & 305.200 & 138.600 & 479.800 & 252.200 & 0.640 \\
\hline B04 & 4710.680 & 3996.259 & 12920.337 & 6060.768 & 0.893 \\
\hline B05 & 1.018 & 1.282 & 3.054 & 0.377 & 1.000 \\
\hline B06 & 954.437 & 1208.703 & 1991.004 & 7278.097 & 1.000 \\
\hline B07 & 3.965 & 5.082 & 13.359 & 3.003 & 0.829 \\
\hline B08 & 14.630 & 16.863 & 44.659 & 14.938 & 0.738 \\
\hline B09 & 11.771 & 6.579 & 22.952 & 15.134 & 0.727 \\
\hline B10 & 364.920 & 244.750 & 923.510 & 1942.935 & 1.000 \\
\hline B11 & 4897.442 & 2787.181 & 11294.607 & 9363.232 & 0.939 \\
\hline B12 & 14.665 & 8.973 & 28.124 & 10.971 & 0.670 \\
\hline B13 & 6.077 & 14.249 & 26.994 & 10.207 & 0.970 \\
\hline B14 & 397.627 & 371.535 & 894.845 & 1902.878 & 0.813 \\
\hline B15 & 661.120 & 830.166 & 2325.128 & 1748.531 & 0.953 \\
\hline B16 & 12.125 & 7.346 & 33.573 & 19.530 & 0.960 \\
\hline B17 & 1222.026 & 1049.479 & 2959.509 & 2651.546 & 0.785 \\
\hline B18 & 931.172 & 838.346 & 2460.798 & 2765.485 & 0.866 \\
\hline B19 & 4070.351 & 2845.498 & 8377.368 & 7726.906 & 0.770 \\
\hline B20 & 3721.233 & 858.463 & 6953.701 & 2779.716 & 1.000 \\
\hline $\mathrm{B} 21$ & 16.137 & 7.080 & 40.771 & 22.126 & 1.000 \\
\hline B22 & 150.706 & 132.504 & 538.754 & 129.956 & 1.000 \\
\hline $\mathrm{B} 23$ & 3857.940 & 2894.374 & 7439.526 & 10239.087 & 0.910 \\
\hline B24 & 7994.808 & 2286.908 & 14156.194 & 11261.820 & 1.000 \\
\hline B25 & 9.689 & 6.975 & 22.432 & 6.032 & 0.756 \\
\hline B26 & 3292.736 & 1953.592 & 7041.164 & 3323.973 & 0.826 \\
\hline B27 & 402.772 & 321.189 & 906.237 & 775.778 & 0.678 \\
\hline B28 & 32.835 & 21.536 & 97.679 & 26.551 & 0.980 \\
\hline B29 & 6.737 & 7.854 & 18.402 & 4.504 & 0.690 \\
\hline B30 & 531.395 & 922.040 & 1672.093 & 1185.165 & 0.815 \\
\hline B31 & 152.510 & 190.361 & 685.374 & 769.898 & 1.000 \\
\hline B32 & 1.925 & 4.581 & 9.163 & 5.274 & 1.000 \\
\hline B33 & 4.889 & 6.737 & 17.402 & 5.082 & 0.840 \\
\hline B34 & 3233.619 & 2527.414 & 7959.733 & 4684.616 & 0.840 \\
\hline B35 & 5169.710 & 5405.975 & 15189.609 & 9830.137 & 0.871 \\
\hline B36 & 6802.566 & 5608.863 & 19958.043 & 15716.893 & 1.000 \\
\hline B37 & 3111.952 & 2126.013 & 6895.572 & 4869.316 & 0.811 \\
\hline B38 & 3600.983 & 1319.711 & 6547.924 & 5116.082 & 0.876 \\
\hline B39 & 7781.754 & 8486.425 & 27514.033 & 14335.679 & 1.000 \\
\hline B40 & 4488.666 & 4531.419 & 12157.913 & 12380.677 & 1.000 \\
\hline B41 & 3188.736 & 1106.154 & 5727.009 & 6194.460 & 1.000 \\
\hline $\mathrm{B} 42$ & 650.830 & 307.959 & 1265.646 & 441.359 & 0.780 \\
\hline
\end{tabular}

